\title{
Rapid Glucocorticoid Receptor-Mediated Inhibition of Hypothalamic-Pituitary-Adrenal Ultradian Activity in Healthy Males
}

\author{
Georgina M. Russell, ${ }^{1}$ David E. Henley, ${ }^{1,2}$ Jack Leendertz, ${ }^{1}$ Jennie A. Douthwaite, ${ }^{1}$ Susan A. Wood, ${ }^{1}$ Adam Stevens, ${ }^{3}$ \\ Wolfram W. Woltersdorf, ${ }^{4}$ Bernard W. M. M. Peeters, ${ }^{5}$ Ge S. F. Ruigt, ${ }^{5}$ Anne White, ${ }^{3}$ Johannes D. Veldhuis, ${ }^{6}$ \\ and Stafford L. Lightman ${ }^{1}$ \\ ${ }^{1}$ Henry Wellcome Laboratories for Integrative Neurosciences and Endocrinology, University of Bristol, Bristol BS1 3NY, United Kingdom, ${ }^{2}$ Faculty of \\ Medicine, Dentistry and Health Sciences, University of Western Australia, Crawley 6009, Western Australia, Australia, ${ }^{3}$ Endocrine Sciences Research \\ Group, Manchester Academic Health Sciences Center, University of Manchester, Manchester M13 9PT, United Kingdom, ${ }^{4}$ University Hospitals Bristol \\ National Health Science Foundation Trust, Department of Laboratory Medicine, Bristol BS2 8HW, United Kingdom, ${ }^{5}$ Schering-Plough, 5340BH 0ss, The \\ Netherlands, and ${ }^{6}$ Endocrine Research Unit, Mayo Clinic College of Medicine, Rochester, Minnesota 55905
}

A complex dynamic ultradian rhythm underlies the hypothalamic-pituitary-adrenal (HPA) circadian rhythm. We have investigated in normal human male subjects the importance, site of action, and receptor-mediated processes involved in rapid basal corticosteroid feedback and its interaction with corticotrophin releasing hormone (CRH) drive. Pro-opiomelanocortin (POMC), ACTH, and cortisol were measured every $10 \mathrm{~min}$ from healthy males during the awakening period or late afternoon using an automated blood sampling system. Mathematical modeling into discrete pulses of activity revealed that intravenous infusion of the synthetic mixed glucocorticoid/ mineralocorticoid agonist prednisolone produced rapid inhibition of ACTH and cortisol pulsatility within 30 min in the morning and afternoon. Any pulse that had commenced at the time of injection was unaffected, and subsequent pulsatility was inhibited. Prednisolone also inhibited ACTH and cortisol secretion in response to exogenous CRH stimulation, inferring rapid feedback inhibition at the anterior pituitary. Circulating POMC peptide concentrations were unaffected, suggesting that the rapid corticosteroid inhibitory effect specifically targeted ACTH secretion from pituitary corticotrophs. Prednisolone fast feedback was only reduced by glucocorticoid receptor antagonist pretreatment and not by mineralocorticoid receptor antagonism, suggesting a glucocorticoid receptor-mediated pathway. The intravenous prednisolone suppression test provides a powerful new tool to investigate HPA abnormalities underlying metabolic and psychiatric disease states.

\section{Introduction}

The hypothalamic-pituitary-adrenal (HPA) axis is characterized by a circadian rhythm, underpinned by a dynamic ultradian rhythm comprising discrete pulses of ACTH and glucocorticoid secretion (Carnes et al., 1989; Windle et al., 1998). HPA activity is tightly controlled via complex regulatory mechanisms of glucocorticoid negative feedback involving signaling networks at the pituitary, hypothalamus, and hippocampus (Watts, 2005). The underlying HPA control mechanism of endogenous cortisol feedback inhibition was first discovered by Moore and Price (1932). Although the basic tenet remains, there is new evidence that ultradian rhythmicity itself is attributable to an oscillatory

Received 0ct. 28, 2009; revised March 10, 2010; accepted March 18, 2010.

This study was supported by Organon BioSciences N.V. (part of Shering-Plough). The Endocrine Sciences Research Group at the University of Manchester is supported by the National Institute for Health Research Biomedical Research funding scheme. We thank Simon Littlewood, Nicola Morgan, Matt Wherlock, and Ashley Bryant for their help.

Correspondence should be addressed to Georgina M. Russell, Henry Wellcome Laboratories for Integrative Neuroscience and Endocrinology, Dorothy Hodgkin Building, University of Bristol, Whitson Street, Bristol BS1 3NY, UK. E-mail: Georgina.Russell@bristol.ac.uk.

DOI:10.1523/JNEUROSCI.5332-09.2010

Copyright $\odot 2010$ the authors $\quad 0270-6474 / 10 / 306106-10 \$ 15.00 / 0$ feedforward-feedback relationship between the pituitary and adrenal gland (Walker et al., 2010). The feedback components of the HPA axis are assumed to act on the two basic processes of peptide synthesis and release (Watts, 2005), mediated via genomic processes through glucocorticoid receptor (GR) and mineralocorticoid receptor (MR) activation (De Kloet et al., 1998). This simple paradigm is probably an oversimplification. It cannot account for multiple sites of action or differential glucocorticoid effects during different physiological conditions and disease states, including depression and the metabolic syndrome (Maddock and Pariante, 2001; Pariante and Miller, 2001). Indeed, there is increasing evidence for nongenomic mechanisms of negative feedback, although differentiation from genomic processes remains controversial (Haller et al., 2008).

Glucocorticoids have been shown to act in rapid nongenomic time domains (fast feedback) (Dallman and Yates, 1969). This has been well documented in rats (Dallman and Yates, 1969; Keller-Wood and Dallman, 1984; Atkinson et al., 2008), and, although there is evidence suggesting a similar mechanism in man (Fehm et al., 1979), it has not been investigated in detail. These rapid effects are important for physiological responses to 
pulsatile glucocorticoid secretion (Stavreva et al., 2009) and may help regulate its characteristic ultradian rhythmicity (Atkinson et al., 2008) via feedforward-feedback loops (Jacobson, 2005; Walker et al., 2010).

Previous studies on negative feedback in humans have predominately used the potent glucocorticoid agonist dexamethasone to inhibit HPA activity over long time domains and have been used in the investigation of affective disorders or Cushing's disease (Schmider et al., 1995; Deuschle et al., 1998; Castro et al., 2003; Grossmann et al., 2004). These studies do not reflect feedback from endogenous pulses of cortisol, which act in a faster time domain and activate both glucocorticoid and mineralocorticoid receptors.

To allow us to determine the dynamics of feedback, we used our human automated blood sampling system (HABS) (Henley et al., 2009) that can painlessly and accurately take frequent venous blood samples for measurement of ultradian hormone secretion under basal conditions. This allowed us to capture rapid inhibition of ACTH and cortisol secretion after intravenous administration of prednisolone. We chose to use the synthetic corticosteroid prednisolone because it has MR and GR binding and activating characteristics similar to cortisol (Grossmann et al., 2004). Used together, the HABS and prednisolone suppression test have allowed, for the first time, the study in healthy males of the characteristics of inhibitory glucocorticoid responses within a rapid time domain, their site and mechanism of action, and importance of endogenous hypothalamic drive.

\section{Materials and Methods}

\section{Participants}

A total of 36 healthy male volunteers (aged 18-34 years, mean of 22 years) were recruited from the students and staff of the University of Bristol (Bristol, UK). Exclusion criteria included (1) body mass index $<18$ or $>30 \mathrm{~kg} / \mathrm{m}^{2}$; (2) medications (including inhalers and steroid creams) except the occasional paracetamol or aspirin in the last 2 months; (3) a history of anxiety, depression, or psychosis; (4) a history of psychosis in a first-degree relative; and (5) allergic reaction or contraindication to any of the study medications used.

This research was conducted in accordance with the Helsinki principles: all participants gave informed written consent before their participation in the study. The study had full ethical approval from the Bath Research Ethics Committee and approval by the Institutional Review Boards of the University Hospitals of Bristol and the University of Bristol. In addition, the study was discussed with the Medicines and Healthcare Products Regulatory Authority and formal approval was deemed unnecessary.

\section{Blood sampling and processing}

Participants were cannulated twice, once in each vein of the antecubital fossa. One cannula was used for collection of venous blood via our HABS described previously by Henley et al. (2009) and the other for the administration of the study reagents. The HABS allows a venous blood sample $(3 \mathrm{ml})$ to be drawn at 10 min intervals via an automated computerized pump. Blood samples were taken for ACTH, cortisol, pro-opiomelanocortin (POMC), and prednisolone levels into EDTA, serum separator tube II, and lithium-heparin tubes and separated within $30 \mathrm{~min}$. Samples for ACTH and POMC were kept on ice until separation. Plasma was divided into aliquots for the different assays and stored at $-80^{\circ} \mathrm{C}$ until analysis.

\section{Assays}

Cortisol levels were determined by RIA (Netria): assay sensitivity, 5 $\mathrm{nmol} / \mathrm{L}$; intra-assay coefficient of variation $(\mathrm{CV}), 5.8 \%$; interassay $\mathrm{CV}$, 9.2\%; and cross reactivity with prednisolone, 6\% (Cortisol RIA kit IM1841; Immunotech). Samples were assayed in duplicate. ACTH levels were processed via chemiluminescent immunometric assay using the Immulite 2000 ACTH kit (Pichler et al., 2004; Raff, 2008): sensitivity, 5 $\mathrm{pg} / \mathrm{ml}$; intra-assay CV, 6.7\%; and interassay CV, 8.2\%. Samples were analyzed singly. Very low ACTH values after prednisolone were documented as $<5 \mathrm{pg} / \mathrm{ml}$ by the analyzer, so for deconvolution analysis, the lower end of the ACTH curve was further calibrated using a polynomial trend line and the equation produced used to deduce ACTH concentration from the counts per second.

Prednisolone levels (combination of two 10-min samples) were measured via HPLC by the Department of Clinical Biochemistry, Royal Brompton Hospital (London, UK) (Robinson et al., 2003). At the prednisolone concentrations being measured, the $\mathrm{CV}$ was $3.4 \%$. The minimum detection limit for the HPLC was $20 \mathrm{nmol} / \mathrm{L}$.

POMC levels were performed using a two-site monoclonal antibodybased ELISA (Rousseau et al., 2007). This had a 100\% cross-reactivity for $\mathrm{POMC}$ and pro-ACTH with a lower limit of detection of $5 \mathrm{pmol} / \mathrm{L}$ and an interassay and intra-assay $\mathrm{CV}$ of $<10 \%$.

\section{Drugs}

Prednisolone sodium succinate (SoluDecortin H) was obtained from Merck, and ovine corticotrophin releasing hormone (oCRH) was from Ferring Pharmaceuticals and prepared according to the instructions of the manufacturer. All drugs were prepared immediately before administration. Prednisolone sodium succinate is the water-soluble form of prednisolone, and cleavage of the ester occurs very rapidly after intravenous injection; free prednisolone levels can be measured $5 \mathrm{~min}$ after the injection (Rose et al., 1981; Boudinot and Jusko, 1986). For the mechanistic studies, mifepristone was purchased from Exelgyn, and spironolactone (nonproprietary) was from Alpharma.

\section{Experimental protocol}

In all studies, six participants attended twice in a blinded randomized order, once for an injection of $10 \mathrm{mg}$ of prednisolone sodium succinate (equivalent to $7.5 \mathrm{mg}$ of prednisolone) over $5 \mathrm{~min}$ and the other for an injection of placebo (equal volume of normal saline) over $5 \mathrm{~min}$. During all testing schedules, the participant was in a separate room to the HABS. The lines connected to the HABS and the line used to deliver the prednisolone, placebo, or other intravenous study medications was passed through a small hatch in the wall above the bed/chair of the participant. If a participant wanted to be disconnected from the HABS to go to the bathroom, this was done between blood samples. During the daytime, participants were free to read, do university work, watch DVDs, etc., and could chose to sit on either a chair or a bed.

Experiment 1: prednisolone-induced feedback inhibition in the early morning. Participants attended the Joint Clinical Research Unit (JCRU) (University Hospitals of Bristol, Bristol, UK) at 10:00 P.M. on the night of testing and cannulated twice. A 30 min HABS acclimatization sequence was performed before lights off at 11:00 P.M. A repeat acclimatization sequence was performed at 4:30 A.M. to ensure that the cannula was still functioning, and the first blood sample was drawn at 5:00 A.M. Ten minute blood samples for the assay of ACTH, cortisol, and POMC were taken throughout the experimental protocol, except for participant $\mathrm{H} 1$, who had 10 min venous blood samples taken for the assay of prednisolone levels instead of POMC to calculate the rate of rise of prednsiolone. Because of the volume of blood required for prednsiolone HPLC, it was not possible to collect a simultaneous sample for the assay of POMC. At 6:02 A.M., participants received $10 \mathrm{mg}$ of prednisolone sodium succinate intravenously or placebo manually over $5 \mathrm{~min}$. Lights switched on at 7:00 A.M., breakfast was served at 8:00 A.M., and the last blood sample was taken at 12:00 P.M.

Experiment 2: prednisolone-induced feedback inhibition in the afternoon/evening. Participants attended the JCRU at 2:00 P.M. and cannulated by 2:30 P.M. At 3:00 P.M., a 30 min HABS acclimatization sequence was performed. At 3:30 P.M., the first blood sample was drawn. Ten minute venous blood samples for the assay of ACTH, cortisol, and POMC levels were taken throughout the experimental protocol, except for participant L1, who had 10 min venous blood samples taken for the assay of prednisolone levels taken instead of POMC as explained in experiment 1. At 4:32 P.M., participants received $10 \mathrm{mg}$ of prednisolone sodium succinate intravenously or placebo manually over $5 \mathrm{~min}$. Dinner was served at 7:30 P.M., and the last blood sample was taken at 10:30 P.M. 
Experiment 3: level of response of prednisolone-induced feedback inhibition. Protocol for experiment 2 was repeated. In addition, participants received two bolus intravenous injections of oCRH at a dose of $1 \mu \mathrm{g} / \mathrm{kg}$ : CRH(1) was given at 4:47 P.M., $10 \mathrm{~min}$ after placebo/prednisolone; $\mathrm{CRH}(2)$ was given $3 \mathrm{~h}$ after $\mathrm{CRH}(1)$, at 7:37 P.M.

Experiment 4: GR and MR contribution to prednisolone-mediated fast feedback. Participants were divided into three subgroups (placebo, mifepristone, and spironolactone), with six participants per group. According to the subgroup, the following combination of additional study medications were administered: oral placebo, mifepristone at $600 \mathrm{mg}$ orally (GR antagonist), or spironolactone at $400 \mathrm{mg}$ orally (MR antagonist). Whichever additional study medications the participants received on their first visit, they also received on their second visit. The order of study subgroup was randomized in a double-blind manner. Participants attended the JCRU at 8:45 A.M. and were cannulated by 9:15 A.M. At 9:30 A.M., a 30 min HABS acclimatization sequence was performed and participants received their study medications. At 10:00 A.M., the first blood sample was drawn. Ten minute venous blood samples for the assay of ACTH and cortisol levels were taken throughout the experimental protocol. Of note, preintervention sampling was extended to $2 \mathrm{~h}$. At 12:02 P.M., participants received $10 \mathrm{mg}$ of prednisolone sodium succinate intravenously or placebo manually over $5 \mathrm{~min}$. Lunch was served at 1:00 P.M., and the last blood sample was taken at 5:00 P.M.

\section{Statistical analyses}

Data are presented as means \pm SE. For all statistical tests, significance at $p<0.05$ was used. Comparison between treatment groups was analyzed using area under the curve (AUC) and all pairwise two-way repeatedmeasures (RM) ANOVA using the Holm-Sidak method. The complete 10 min data were separated into smaller timeframes. Results presented are for the absolute values (un-normalized) and normalized (average preintervention activity) data.

ACTH and cortisol concentration time series were analyzed using a recently developed automated deconvolution method, which was mathematically verified by direct statistical proof and empirically validated using hypothalamic-pituitary sampling and simulated pulsatile time series (Keenan et al., 2001, 2003). The MATLAB-based algorithm first detrends the data and normalizes concentrations to the unit interval $[0,1]$ (Keenan et al., 2005). Second, the program creates multiple successive potential pulse-time sets, each containing one fewer burst via a smoothing process (a nonlinear adaption of the heat-diffusion equation). Third, a maximum-likelihood expectation estimation method computes all secretion and elimination parameters simultaneously conditional on each of the candidate pulse-time sets. Deconvolution parameters comprise basal secretion $\left(\beta_{0}\right)$, two half-lives $\left(\alpha_{1}, \alpha_{2}\right)$, secretory-burst mass $\left(\eta_{0}, \eta_{1}\right)$, random effects on burst mass $\left(\sigma_{\mathrm{A}}\right)$, measurement error $\left(\sigma_{\varepsilon}\right)$, and a three-parameter flexible gamma secretory-burst waveform $\left(\beta_{1}, \beta_{2}, \beta_{3}\right)$. For ACTH, the fast half-life was represented as $3.5 \mathrm{~min}$, constituting $37 \%$ of the decay amplitude (Iranmanesh et al., 1993). The slow half-life was represented as an unknown variable between 14 and $35 \mathrm{~min}$. For cortisol, the rapid half-life was fixed at $2.4 \mathrm{~min}$ ( $37 \%$ of decay amplitude), and the slow half-life was estimated as a variable ( $56 \mathrm{~min}$ ) within the expected range of $40-85 \mathrm{~min}$ (Bright, 1995). All candidate pulse-time sets were deconvolved. Statistical model selection was then performed to distinguish among the independently framed fits of the multiple candidate pulse-time sets using the Akaike information criterion (Akaike, 1974). Observed interpulse intervals were described by a twoparameter Weibull process (more general form of a Poisson process, which uncouples the mean from the variance). The parameters (and units) are frequency (number of bursts per total sampling period, $\lambda$ of Weibull distribution), regularity of interpulse intervals (unitless $\gamma$ of Weibull), slow halflife (minutes), basal and pulsatile secretion rates (concentration units/ session), mass secreted per burst (concentration units), and waveform shape [mode or time delay to maximal secretion after objectively estimated burst onset (minutes)]. To calculate average pulse mass according to the timeframe of the first $2 \mathrm{~h}(0-120 \mathrm{~min})$ and the last $3 \mathrm{~h}(121-360 \mathrm{~min})$ postintervention start time, pulsatile activity included was based on predicted putative pulse onset occurring within the allotted timeframe. Results presented are for the absolute values and normalized (average placebo pulse mass used as surrogate marker for baseline activity for experiments 1-3, baseline prein-
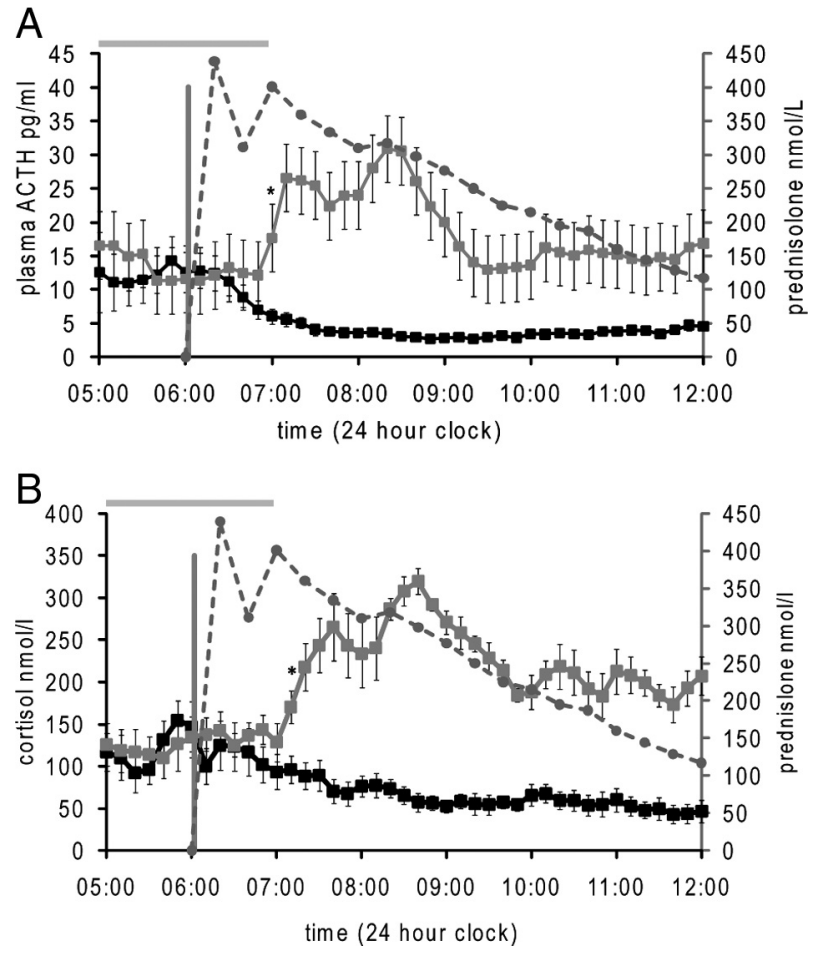

Figure 1. Six healthy male subjects sampled at $10 \mathrm{~min}$ intervals for ACTH $(\boldsymbol{A})$ and cortisol ( $\boldsymbol{B})$ presented as group means \pm SEM. Subjects attended twice and were given either an intravenous injection of $10 \mathrm{mg}$ of prednisolone sodium succinate (black squares) or placebo (gray squares) over 5 min at 6:02 A.M. (shaded vertical line) (period of lights off indicated by shaded horizontal line). Prednisolone (gray circle dotted line) had a rate of rise of 21 $\mathrm{nmol} \cdot \mathrm{L}^{-1} \cdot \mathrm{min}^{-1}$ in the first $20 \mathrm{~min}$. A steady-state concentration was then reached, and levels began to decline 40 min later. ${ }^{*}$ denotes first significant difference between the prednisolone and placebo groups.

tervention activity for experiment 4) data. For the afternoon studies deconvolution analysis data, subject L1 was excluded as a result of a stress response on his first visit (placebo intervention day). Stress response exclusion criteria included a double than or greater difference in preintervention pulse mass between placebo and prednsiolone subgroups. Comparisons within and between treatment groups were analyzed using a two-tailed paired Student's $t$ test. If there was no detectable activity after intervention, the minimal detectable pulse mass for the program was assigned ( $1 \mathrm{pg} / \mathrm{ml} \mathrm{ACTH}$ and $3 \mathrm{nmol} / \mathrm{L}$ cortisol).

For the participants who received the prednisolone intervention during an interpulse interval, time to inhibition was calculated as the time taken from the start of prednisolone intervention to the next putative pulse onset as estimated by deconvolution analysis. Evidence of partial inhibition was taken as $<50 \%$ of baseline activity and complete inhibition as $<30 \%$ of baseline activity.

To quantitate the orderliness or regularity of consecutive serum POMC levels over the sampling period, approximate entropy (ApEn) was used. Normalized ApEn parameters of $m=1$ (run length) and $r=$ $20 \%$ (tolerance window) of each series SD were used. The distribution of empirically "random" (maximal) ApEn values were determined by 1000 random shuffles of ordered sample values within each time series. Therefore, the ApEn of each "observed" (unshuffled) time series was also evaluated as a mean ratio of observed-to-random ApEn (Veldhuis et al., 2001). Higher absolute ApEn values denote greater relative randomness of hormone secretion pattern (Pincus and Keefe, 1992).

\section{Results}

Experiment 1: prednisolone inhibition of ACTH and cortisol secretion during the morning

Ten minute sampling (Fig. 1) of acute administration of prednisolone produced a significant decline in ACTH values within 60 

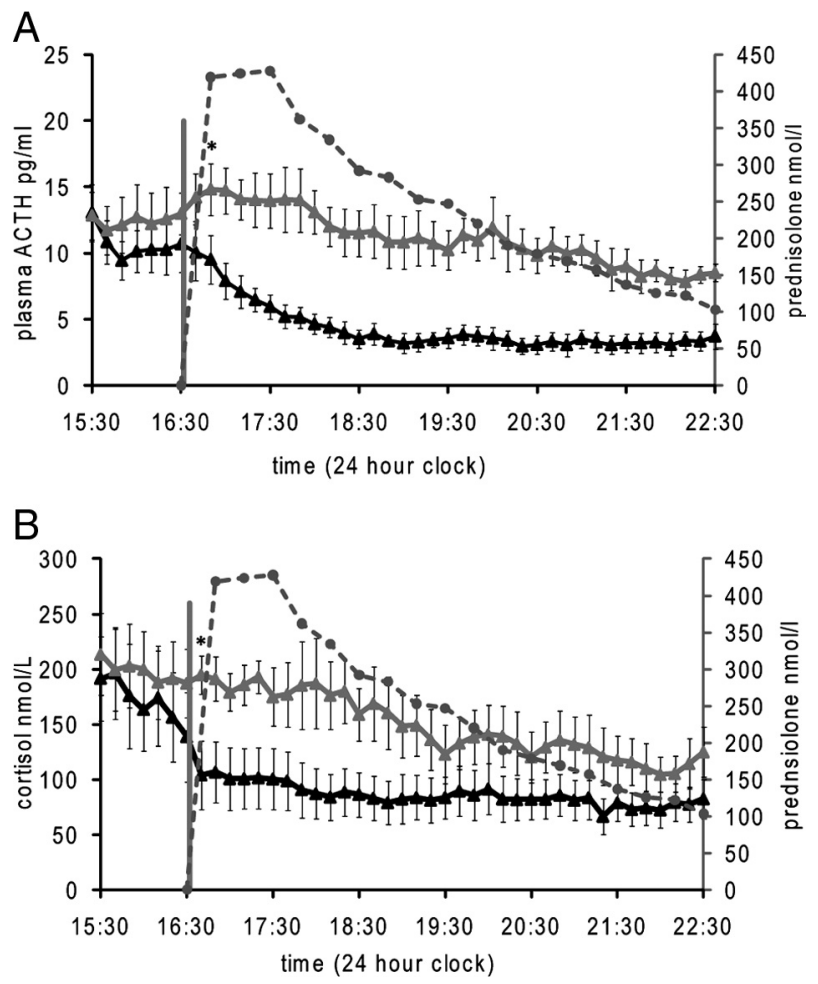

Figure 2. Six healthy male subjects sampled at 10 min intervals for ACTH ( $\boldsymbol{A}$ ) and cortisol ( $\boldsymbol{B})$ presented as group means \pm SEM. Subjects attended twice and were given either an intravenous injection of $10 \mathrm{mg}$ of prednisolone sodium succinate (black triangles) or placebo (gray triangles) over $5 \mathrm{~min}$ at 4:32 P.M. (shaded vertical line). Prednisolone (gray circle dotted line) achieved a rate of rise of $20 \mathrm{nmol} \cdot \mathrm{L}^{-1} \cdot \mathrm{min}^{-1}$ in the first $20 \mathrm{~min}$. A steady-state concentration was then reached, and levels began to decline 40 min later. ${ }^{*}$ denotes first significant difference between the prednisolone and placebo groups.

$\min (p=0.001, \mathrm{RM}-\mathrm{ANOVA})$ and for cortisol within $70 \mathrm{~min}$ $(p<0.001$, RM-ANOVA) on both absolute values and normalized data. There was no significant difference in preintervention ACTH and cortisol values.

ACTH and cortisol pulsatility were significantly reduced after prednisolone $(p<0.01)$ (supplemental Table $1 A, B$, available at www.jneurosci.org as supplemental material), and there was a significant reduction in ACTH basal secretion ACTH $(p<0.05)$ (supplemental Table $1 A$, available at www.jneurosci.org as supplemental material). Overall cortisol basal secretion was also reduced but was not significantly apparent after deconvolution (supplemental Table $1 B$, available at www.jneurosci.org as supplemental material). After prednisolone, there was a significant reduction in average ACTH and cortisol pulse mass within $2 \mathrm{~h}$ in the prednisolone group compared with baseline activity $(\mathrm{ACTH}$, $p<0.001$ absolute values/normalized; cortisol, $p<0.01$ absolute values/normalized) and placebo at a similar timeframe $(p<0.05$ absolute values, $p<0.001$ normalized; and $p<0.01$ absolute values, $p<0.05$ normalized, respectively). The degree of inhibition continued until the end of the sampling period (supplemental Tables $2 A-C, 3 A-C$, available at www.jneurosci.org as supplemental material). In the placebo group, there was a significant increase in average pulse mass for ACTH corresponding with the awakening period ( $p<0.01$ absolute values; $p<0.001$ normalized). From $2 \mathrm{~h}$ after placebo intervention until the end of the sampling period, the average ACTH pulse mass was significantly smaller than baseline activity ( $p<0.05$ absolute values; $p<0.001$ normalized) but still greater than the corresponding prednisolone group ( $p=0.005$ absolute values; $p<0.005$ normalized).
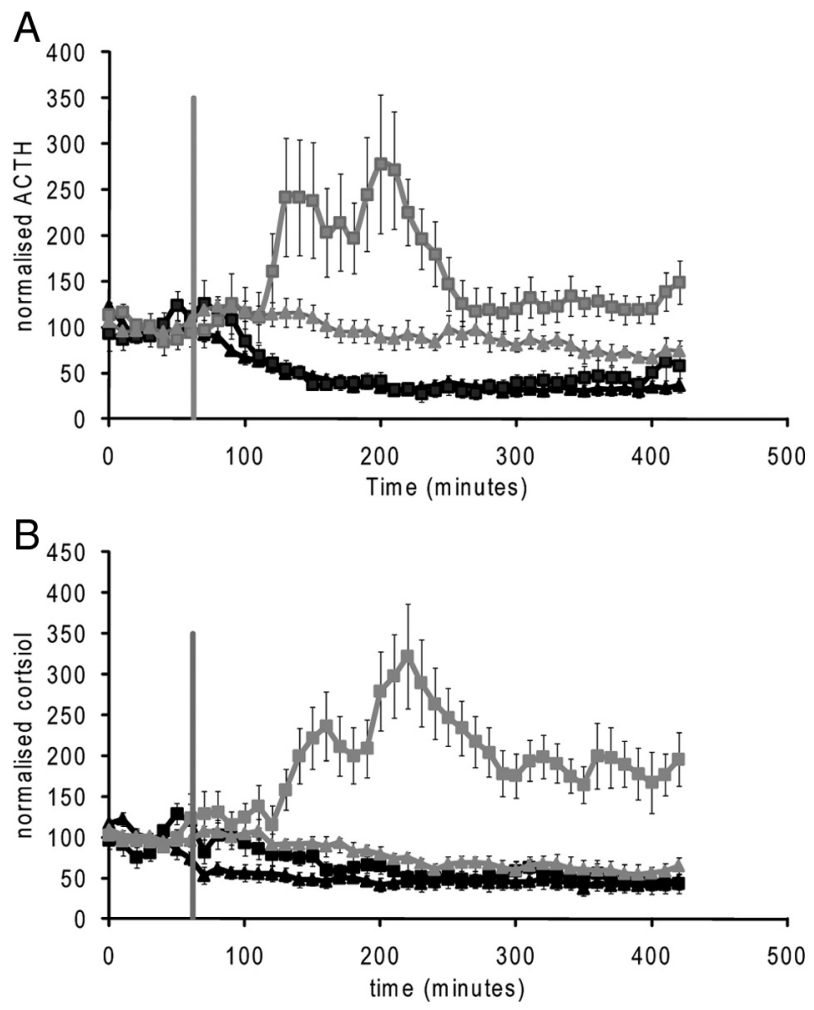

Figure 3. Comparison of morning (squares) and afternoon (triangles) studies. Six healthy male subjects sampled at 10 min intervals for ACTH $(\boldsymbol{A})$ and cortisol $(\boldsymbol{B})$ presented as group normalized means \pm SEM. Subjects attended twice and were given either an intravenous injection of $10 \mathrm{mg}$ of prednisolone sodium succinate (black) or placebo (gray) over $5 \mathrm{~min}, 62 \mathrm{~min}$ into sampling (vertical line).

For cortisol, in the placebo intervention group, the average pulse mass in the first $2 \mathrm{~h}$ after placebo intervention (corresponding with the awakening rise) was significantly larger than the remainder of the sampling period ( $p<0.05$ absolute values; $p<$ 0.005 normalized). There was no difference from baseline for cortisol for either the average pulse mass in the first $2 \mathrm{~h}$ after intervention or the remainder of the sampling period.

\section{Experiment 2: prednisolone inhibition on ACTH and cortisol secretion during the late afternoon}

Acute administration of prednisolone to normal volunteers in the late afternoon (Fig. 2) produced a significant decline within 10 min for ACTH $(p<0.005$ absolute values; $p<0.001$ normalized, RM-ANOVA) and also for cortisol levels (absolute values nonsignificant, $p<0.001$ normalized, RM-ANOVA). There was no difference in preintervention sampling.

There was a significant reduction in ACTH total pulsatility and basal secretion after prednisolone $(p<0.05)$, although no such difference was detectable for cortisol (supplemental Table $1 A, B$, available at www.jneurosci.org as supplemental material). After prednisolone, there was a significant reduction in ACTH and cortisol pulse mass compared with the placebo intervention (ACTH, $p<0.05$ absolute values, $p=0.001$ normalized; cortisol normalized data, $p<0.05)$ and baseline activity (ACTH, $p<0.01$ absolute values, $p<0.001$ normalized; and cortisol, $p=0.05$ absolute values, $p<0.001$ normalized) within $2 \mathrm{~h}$ (supplemental Tables 4, 5, available at www.jneurosci.org as supplemental material). This degree of inhibition continued until the end of the sampling period for ACTH only. For cortisol, two of the subjects regained their endogenous pulsatility; consequently, there was no 
statistically significant difference in average pulse mass between the placebo and prednisolone intervention groups after $2 \mathrm{~h}$.

The placebo group, as expected at this time of day, showed a general decline in ACTH $(p<0.05$ absolute values; $p<$ 0.005 normalized $)$ and cortisol $(p<0.05$ normalized) levels over the sampling period compared with baseline sampling.

\section{Direct comparison of morning and} afternoon responses to corticosteroid On examination of the normalized ACTH and cortisol data, there were no differences in the responses elicited by prednisolone at the two times of day as shown through assessment of AUC, RM-ANOVA, and average pulse mass according to timeframe (Fig. 3). In the placebo intervention groups, the morning studies produced a greater normalized AUC for ACTH $(p<$ $0.05)$ and cortisol $(p<0.001)$. Normalized average pulse mass confirmed a greater ACTH pulse mass in the first $2 \mathrm{~h}$ after intervention, corresponding to the awakening response. This did not reach statistical significance for cortisol. Basal ACTH and cortisol secretion in the placebo intervention groups was similar at both times of day, as was the reduced ACTH basal secretion seen after prednisolone.

\section{Interaction of glucocorticoid inhibition} on ACTH and cortisol with phase of endogenous ultradian rhythm

Hormone pulses that were initiated at the time of prednisolone injection continued in a classical manner (Fig. 4A,B). Subsequent pulsatile activity was either inhibited (typically $<30 \%$ by mass) and appeared delayed or was absent. Subjects may or may not have exhibited recovery of endogenous pulsatility by the end of the sampling period. Consequently, it was difficult to interpret when pulsatility was first affected.

If, however, the injection of prednisolone was given during the interpulse period (Fig. $4 C$ ), the subsequent pulse occurred at a time that appeared appropriate but was smaller than expected, and the degree of inhibition was related to the length of time after prednisolone and the putative pulse onset time; the greater the time from prednisolone treatment to pulse onset, the greater the degree of inhibition. Partial inhibition ( $50 \%$ by mass) was first documented at $8 \mathrm{~min}$ after prednisolone and complete inhibition by 10-12 min after prednisolone for both morning and afternoon studies. Pearson's product moment correlation (Fig. 5) confirmed a negative correlation between time to inhibition and pulse mass expressed as a percentage of baseline for ACTH $(r=$ $-0.9, p<0.01, n=6$ ). A similar negative correlation existed for cortisol but did not reach significance $(r=-0.7, p$ value was nonsignificant, $n=5$ ).

\section{Experiment 3: prednisolone inhibition of ACTH and cortisol} secretion after oCRH stimulation

Prednisolone in combination with oCRH produced a significant decline in ACTH values within $50 \mathrm{~min}(p<0.001$, RM-ANOVA)
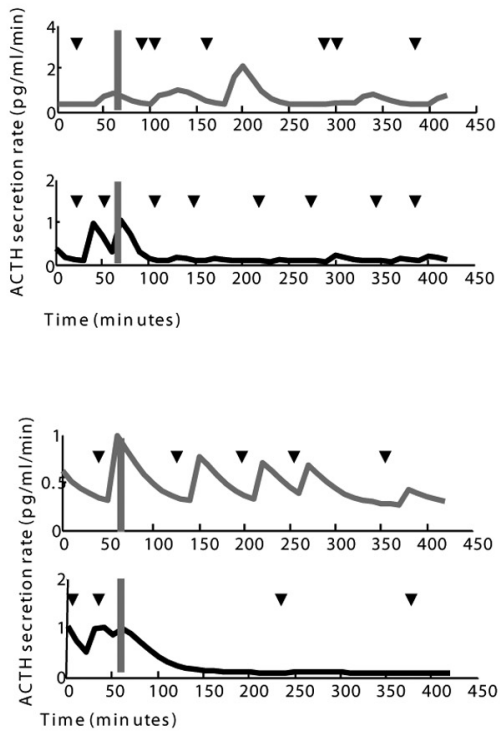
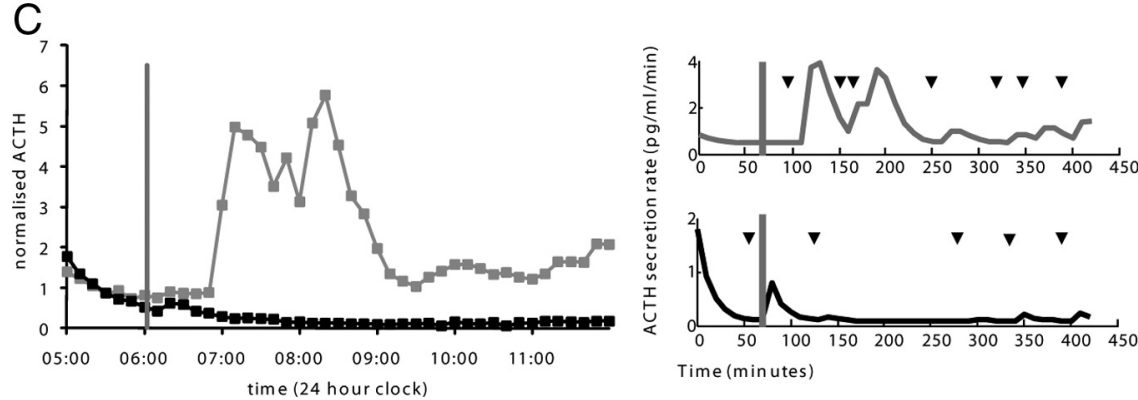

Figure 4. Examples of participants' ACTH profiles and corresponding mathematically modeled pulsatile activity after placebo (brednisolone (black) in the morning (squares) and afternoon (triangles) are shown. In $\boldsymbol{A}$, the participant received prednisolone at the beginning of a pulse of activity, in $\boldsymbol{B}$ at the end of an endogenous pulse, and $\boldsymbol{C}$ during an interpulse interval. Estimated putative pulse onsets are documented by black triangles above the time line of the mathematically modeled profiles.

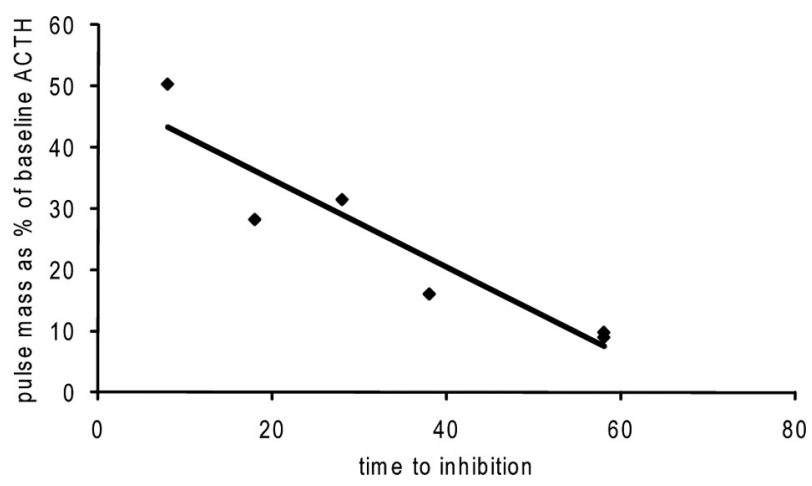

Figure 5. Relationship between time to inhibition (minutes) after prednisolone and degree of inhibition (expressed as percentage of baseline average pulse mass) for ACTH pulsatility $(r=-0.9, p<0.01, n=6)$.

for both absolute and normalized values compared with placebo and oCRH. For cortisol, the inhibition occurred within $10 \mathrm{~min}$ for the absolute values $(p<0.001)$ and within 70 min for normalized cortisol $(p<0.001)$ (Fig. 6). There was no difference in preintervention sampling.

For both ACTH and cortisol, acute intravenous injection of prednisolone inhibited the response to both $\mathrm{CRH}(1)(p<0.05$ and $p=0.05)$ and $\mathrm{CRH}(2)(p=0.01$ and $p<0.05)$ injections 
A
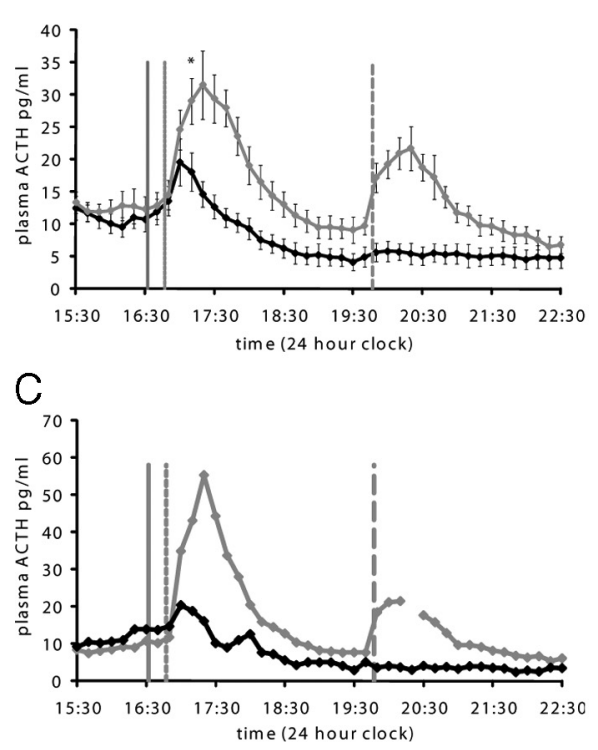

B
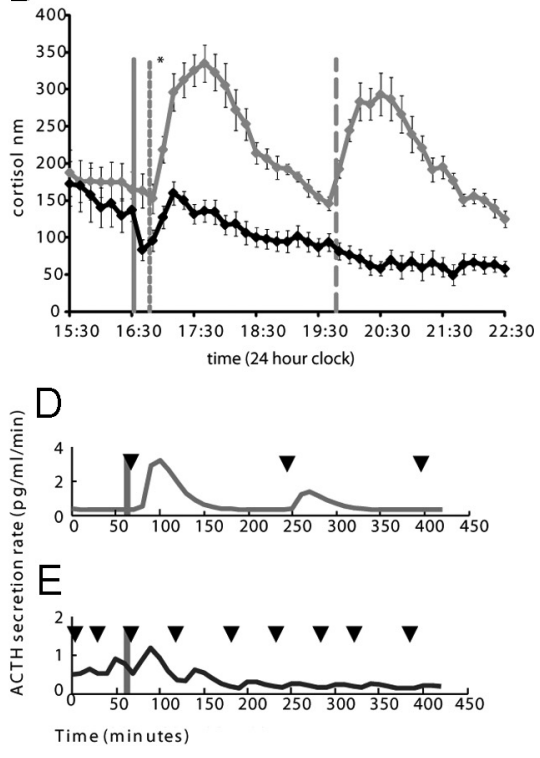

Figure 6. Six healthy male subjects sampled at 10 min intervals for ACTH $(\boldsymbol{A})$ and cortisol $(\boldsymbol{B})$ presented as group means \pm SEM. Subjects attended twice and were given either an intravenous injection of $10 \mathrm{mg}$ of prednisolone sodium succinate (black diamonds) or placebo (gray diamonds) over 5 min at 4:32 P.M. (shaded line) and an injection of oCRH 10 min (gray dotted line) and $3 \mathrm{~h}$ (gray dashed line) later at the dose of $1 \mu \mathrm{g} / \mathrm{kg}$. ${ }^{*}$ denotes first significant difference between the prednisolone and placebo interventions with serial oCRH stimulation. An example of a subject's ACTH profile after placebo and prednisolone is shown in $C$. The corresponding mathematically modeled pulsatile activity is shown for placebo $(\boldsymbol{D})$ and prednisolone $(\boldsymbol{E})$ interventions, and putative pulse onsets denoted by black triangles above the time line.

more than placebo intervention. Prednisolone reduced the response to $\mathrm{CRH}(2)$ more than $\mathrm{CRH}(1)$ for both ACTH and cortisol $(p<0.05)$. After placebo, the response to $\mathrm{CRH}(2)$ was smaller than CRH(1) for ACTH only ( $p=0.05$ ) (supplemental Table $6 B-D$, available at www.jneurosci.org as supplemental material). Total pulsatility was significantly reduced in the prednisolone intervention arm for ACTH and cortisol ( $p=0.01$ and $p<0.05$ respectively) (supplemental Table $6 A$, available at www. jneurosci.org as supplemental material).

On examination of putative pulse onset times, the injection of oCRH synchronized the subjects by inducing a pulse of activity. There was evidence of inhibition of ACTH pulsatile activity in all subjects within $20 \mathrm{~min}$ and for cortisol within $30 \mathrm{~min}$ for all but one subject.

ACTH basal secretion was reduced after prednisolone $(p<$ 0.01) (supplemental Table 6A, available at www.jneurosci.org as supplemental material). The ACTH basal secretion after placebo and prednisolone in the oCRH studies was not statistically different from the corresponding morning and afternoon studies. For cortisol, there was a trend toward reduced basal secretion after prednisolone, and this did not reach significance (supplemental Table $6 A$, available at www.jneurosci.org as supplemental material).

\section{Circulating POMC concentrations}

POMC, the precursor of ACTH, was secreted into the circulation with concentrations of 5-25 pmol/L (mean of $15 \mathrm{pmol} / \mathrm{L}$ ) for the morning studies, $17-100 \mathrm{pmol} / \mathrm{L}$ (mean of $63 \mathrm{pmol} / \mathrm{L}$ ) for the afternoon studies, and $17-130 \mathrm{pmol} / \mathrm{L}$ (mean of $43 \mathrm{pmol} / \mathrm{L}$ ) for the oCRH stimulation studies. Based on AUC assessment, the POMC levels were significantly lower in the morning compared with the afternoon $(p<0.001$, two-tailed unpaired Student's $t$ test). There was no inhibition of POMC secretion with pred- nisolone during any timeframe measured, independent of time of day as assessed by AUC (Fig. 7).

Interestingly, POMC was not stimulated by oCRH over $180 \mathrm{~min}$ and even after repeated CRH stimulation. Mean POMC concentrations were $43 \mathrm{pmol} / \mathrm{L}$ after oCRH, which did not differ from placebo concentrations in the afternoon.

To assess whether POMC had any significant pulsatility, the mean absolute ApEn value was calculated. For POMC, this was $1.03 \pm 0.07$ with a ratio (to shuffled) of 1.08 for the morning studies, $1.14 \pm 0.07$ with a ratio (to shuffled) of 1.20 for the afternoon studies, and $1.12 \pm$ 0.06 with a ratio (to shuffled) of 0.91 for the oCRH-stimulated studies. This indicated that, in all of the studies over the timeframe measured, the POMC concentration series was $\sim 100 \%$ toward random, indicating no significant pulsatility.

Experiment 4: GR and MR contribution to prednisolone-mediated fast feedback Pretreatment with spironolactone did not modify the rapid inhibitory response to prednisolone intervention, nor were there any significant differences between the spironolactone and placebo groups within any time domain using RM-ANOVA (Fig. 8). Pretreatment with oral placebo was similar to previous experiments (Figs. 1, 2, 8, 9). Furthermore, deconvolution analysis revealed a similar rapid pattern of inhibition of ACTH and cortisol pulsatility with no statistically significant differences in normalized average pulse mass according to timeframe between the prednisolone intervention responses of the spironolactone and placebo groups. Because of a lack of preintervention data, the placebo intervention results could not be analyzed (supplemental Tables $7 B, C, 8 B, C$, available at www.jneurosci.org as supplemental material). There were no statistically significant differences seen in ACTH and cortisol basal secretion compared with placebo preintervention with a reduction in ACTH basal secretion $(p<0.005)$ only (supplemental Tables $7 A, 8 A$, available at www.jneurosci.org as supplemental material).

In contrast to the lack of effect with spironolactone, pretreatment with mifepristone did both reduce and delay the ACTH inhibition after prednisolone ( $p<0.05$ normalized, RM-ANOVA). Specifically, in the mifepristone group (Fig. 9), acute administration of prednisolone produced a significant decline in ACTH levels only after $2 \mathrm{~h}(p<0.05$ absolute; $p<0.001$ normalized, RM-ANOVA) and after $3 \mathrm{~h}$ for cortisol levels $(p<0.01$ absolute; $p<0.05$ normalized, RM-ANOVA). A similar pattern could be seen for normalized cortisol data but did not reach statistical significance. There were no statistically significant differences between normalized ACTH and cortisol (RM-ANOVA) in those pretreated with mifepristone and placebo after placebo intervention.

Deconvolution analysis confirmed the loss of rapid inhibition by prednisolone after mifepristone treatment (supplemental Tables $7 B, C, 8 B, C$, available at www.jneurosci.org as supplemental material). Prednisolone produced a significant reduction in average ACTH pulse mass only after $2 \mathrm{~h}$ compared with baseline activity (ACTH, $p=0.001$ normalized), and no difference was 

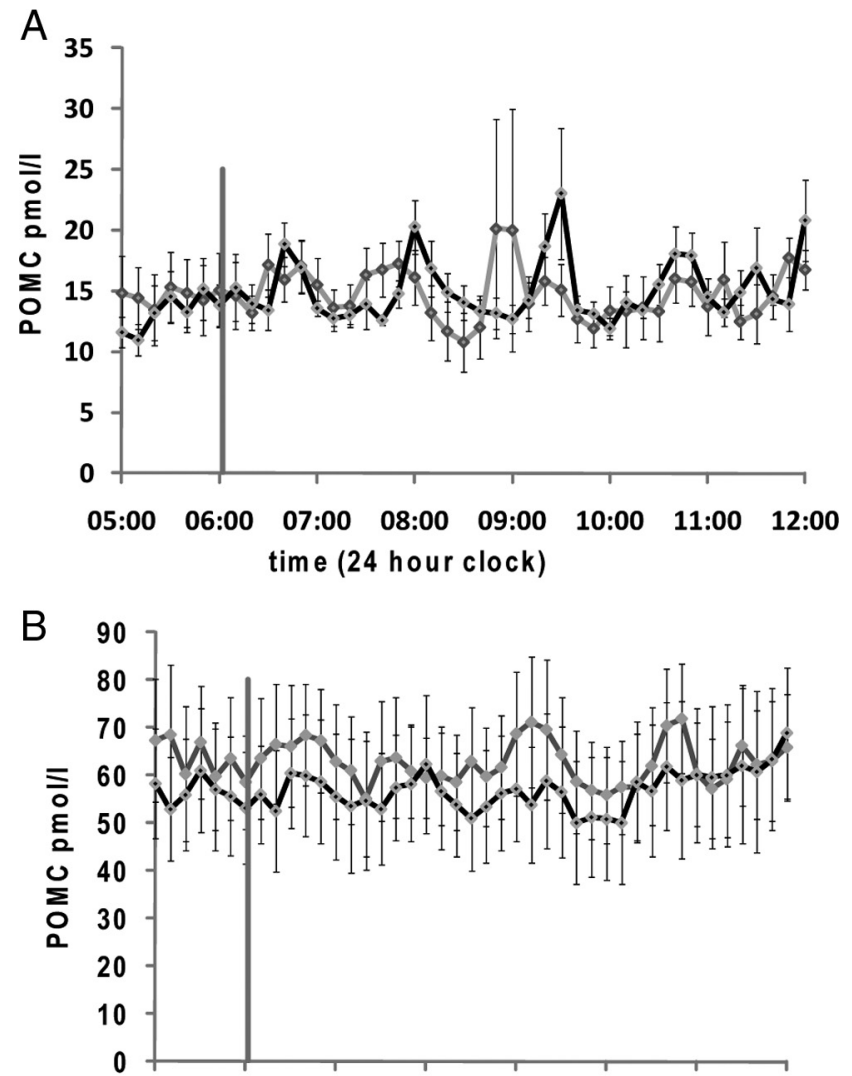

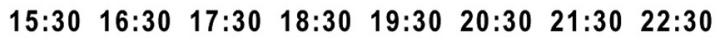
time (24 hour clock)

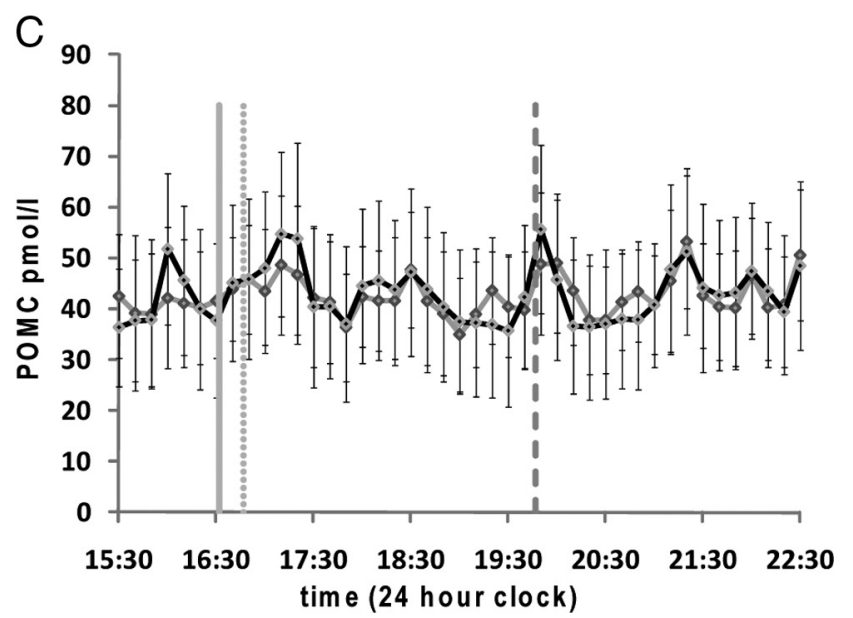

Figure 7. POMC, the precursor of ACTH, was measured at the same time as ACTH in all subjects, with placebo (gray diamonds) and prednisolone (black squares) given over $5 \mathrm{~min}$ (shaded line). A shows morning sampling, $\boldsymbol{B}$ shows afternoon sampling, and $($ shows afternoon sampling with exogenous oCRH stimulation $10 \mathrm{~min}$ after intervention (dotted line) and $3 \mathrm{~h}$ later (dashed line). Results are presented as group means \pm SEM.

seen compared with placebo intervention over a similar timeframe. There were no statistically significant differences seen in cortisol average pulse mass (absolute and normalized). Indeed, there was a trend to a larger ACTH and cortisol pulse mass in the first $2 \mathrm{~h}$ after prednisolone intervention ( $p=0.07$ and $p=0.08)$. This reached statistical significance after $2 \mathrm{~h}$ for ACTH $(p<$ $0.05)$, and the trend continued for cortisol $(p=0.06)$. After mifepristone and placebo intervention, there was no significant
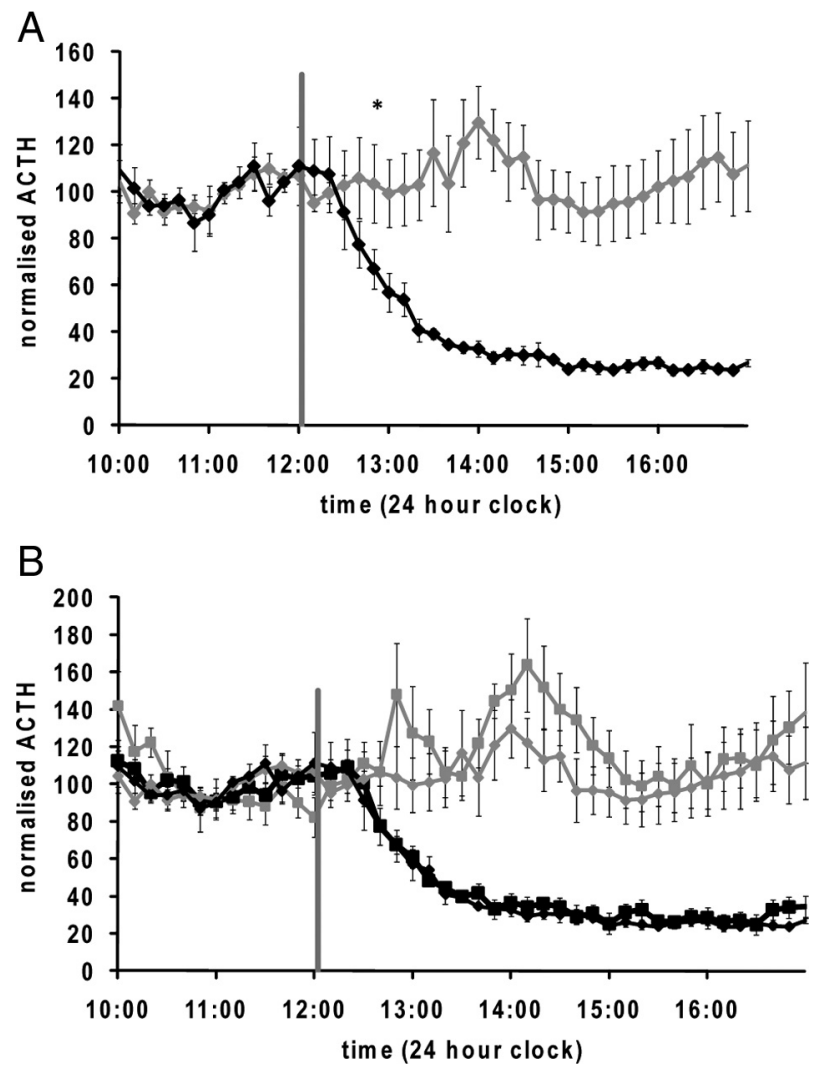

Figure 8. 6 healthy male subjects sampled at 10 min intervals for ACTH and cortisol. Data presented are group normalized ACTH means $\pm \operatorname{SEM}(\boldsymbol{A})$. Subjects attended twice and were given oral spironolactone at 9:30 A.M., followed by either an intravenous injection of $10 \mathrm{mg}$ of prednisolone sodium succinate (black diamonds) or placebo (gray diamonds) over $5 \mathrm{~min}$ at 12:02 P.M. (shaded vertical line). * denotes first significant difference between the prednisolone and placebo interventions. $\boldsymbol{B}$ shows comparison of normalized mean \pm SEM ACTH responses after placebo (gray) and prednisolone (black) intervention with (diamonds) and without (squares) spironolactone.

change in average pulse mass (absolute and normalized) for ACTH or cortisol throughout the sampling period.

Additionally, mifepristone pretreatment attenuated the reduction seen in basal ACTH secretion after prednisolone, with mifepristone pretreatment resulting in statistically significant larger basal ACTH secretion ( $p=0.01$ ) (supplemental Table 7A, $8 A$, available at www.jneurosci.org as supplemental material).

\section{Discussion}

Fast feedback was first described in the rat under conditions of stress by Dallman and Yates in 1969 and later characterized as part of a two-phase pattern of inhibition of HPA activity (KellerWood and Dallman, 1984). Similar studies in man were performed in hypoadrenal patients off replacement therapy who also showed a similar two-phase period of feedback inhibition (Fehm et al., 1979). The human inhibitory time course shows great variability and has been described as anything between 15 and 60 min (Reader et al., 1982; Posener et al., 1997; Boscaro et al., 1998). Because of this variability, there has been uncertainty as to whether the mechanisms underlying the feedback differed from that in the animal model.

In our studies, we have used the HABS (Henley et al., 2009) to enable us to do a full investigation of the patterns of HPA activity under basal and feedback inhibition conditions. We used prednisolone to induce feedback inhibition because it activates both MR 

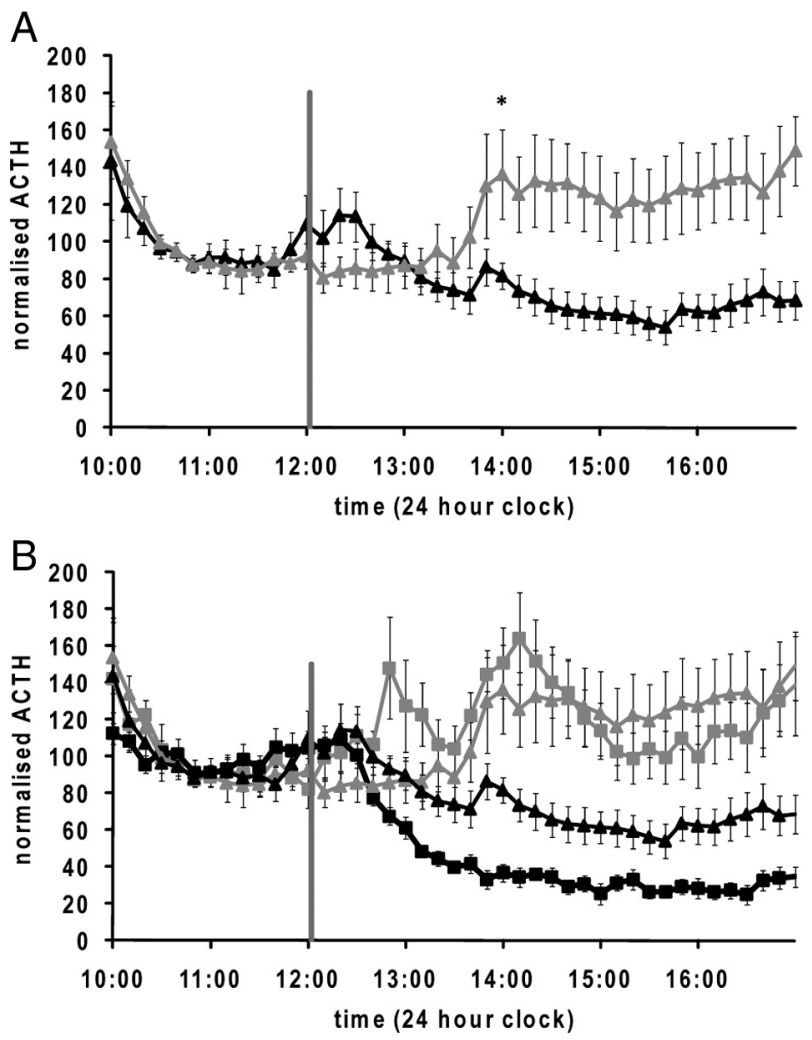

Figure 9. $A$, Group normalized ACTH means \pm SEM for six healthy male subjects who attended twice and were given oral placebo at 9:30 A.M., followed by either an intravenous injection of $10 \mathrm{mg}$ of prednisolone sodium succinate (black triangles) or placebo (gray triangles) over 5 min at 12:02 P.M. (shaded vertical line). ${ }^{*}$ denotes first significant difference between the prednisolone and placebo interventions. $\boldsymbol{B}$ shows comparison of normalized mean \pm SEM ACTH responses after placebo (gray) and prednisolone (black) with (triangles) and without (squares) mifepristone.

and GR (Grossmann et al., 2004) and allowed us to measure HPA responses without cross-reactivity with endogenous cortisol.

Resolving the ACTH and cortisol profiles into discrete pulses of activity by deconvolution analysis revealed very rapid feedback inhibition. Indeed, the pattern of inhibition was identical to the rodent model (Atkinson et al., 2008) in which feedback inhibition showed a distinct relationship with the phase of the endogenous ultradian rhythm. We find that any pulse that has commenced at the time of prednisolone injection was completed, whereas any subsequent pulsatile activity was significantly smaller $(<30 \%)$. If, however, prednisolone was given during the interpulse period, subsequent pulsatile activity occurred at an appropriate time but pulses were smaller than expected. Interestingly, the longer the time after prednisolone to pulse onset, the greater the degree of inhibition, with partial inhibition (50\% of baseline activity) at 8 min and complete inhibition ( $<30 \%$ of baseline activity) by 10-12 min. This rapid inhibition was present for ACTH and cortisol and shows for the first time that, in the basal state, humans show rapid HPA negative feedback.

This relationship of feedback response with the phase of the endogenous ultradian rhythm explains the marked variation in previously documented speed and duration of feedback inhibition (Reader et al., 1982; Posener et al., 1997; Boscaro et al., 1998). Because pulsatility is not synchronized between subjects and the interpulse interval for ACTH is $\sim 40$ min (Veldhuis et al., 1990) and for cortisol is 80-110 min (Follenius et al., 1987; Veldhuis et al., 1989), prednisolone-induced inhibition may occur from minutes to $1 \mathrm{~h}$ for ACTH or up to $2 \mathrm{~h}$ for cortisol, emphasizing the importance of adjuvant methods such as mathematical modeling when examining "noisy" datasets.

It is also interesting to note that, as in the animal model (Atkinson et al., 2008), some of our subjects demonstrated recovery of endogenous cortisol pulsatility after approximately $5 \mathrm{~h}$. This only occurred in the afternoon, suggesting that, although rapid inhibition itself was independent of time of day and consequently CRH drive, response duration does depend on chronobiology. Because this recovery was seen for cortisol rather than ACTH, it suggests involvement of a non-ACTH input such as the splanchnic nerve (Ulrich-Lai et al., 2006) or intra-adrenal clock (Son et al., 2008).

Deconvolution analysis also revealed that prednisolone resulted in reduced basal secretion of ACTH. Because deconvolution analysis assumes a steady-state basal release, it is not possible to ascertain whether inhibition of basal, as opposed to pulsatile, secretion was similarly rapid or a genomically mediated gradual decline. Interestingly, cortisol basal secretion was unaffected.

Serial injections of oCRH synchronized pulsatility allowed estimation of rapidity of feedback response to prednisolone in all participants. Inhibition of the $\mathrm{CRH}$ response occurred within $<20 \mathrm{~min}$ for ACTH and typically $<30 \mathrm{~min}$ for cortisol. Complete inhibition of CRH stimulation occurred after the second injection of oCRH $3 \mathrm{~h}$ after prednisolone. These observations concur with animal work (Zimmermann and Critchlow, 1969, 1972). This is the first evidence for rapid feedback at the level of the anterior pituitary gland in man and fits with early in vitro (Sayers and Portanova, 1974) and in vivo (Jones et al., 1977) studies in the rat. In the placebo wing of this study, the second oCRH injection resulted in a blunted response as has been documented previously (Young et al., 2004), although basal ACTH secretion was unaffected, suggesting an action of $\mathrm{CRH}$ on the pulsatile mechanism only.

Although there is rodent data suggesting that fast feedback can occur independent of protein synthesis (Keller-Wood and Dallman, 1984) without modification of POMC processing to ACTH (Roberts et al., 1982), there is no equivalent data in man. We addressed this by the measurement of the release of unprocessed POMC for which we found no evidence of pulsatile release, suggesting that it was not released through the normal regulatory secretory pathway. POMC levels were higher in the afternoon than in the morning, which may be a reflection of differential processing of POMC into ACTH. Circulating POMC was unaffected by either prednisolone or oCRH, further reinforcing the concept that POMC is released from pituitary corticotrophs by a constitutive pathway insensitive to corticosteroid feedback (Pritchard and White, 2007). The fast inhibitory feedback of glucocorticoids in man therefore appears to be selective for the regulated release mechanism.

Although pretreatment with the MR antagonist spironolactone had no effect on the inhibitory response to prednisolone, the selected GR antagonist mifepristone resulted in a significant reduction in the inhibition of ACTH and cortisol levels and pulse mass. Most marked was the fact that, after mifepristone, there was no significant feedback inhibition from prednisolone for $2 \mathrm{~h}$, and there was complete inhibition of the prednisolone-induced reduction of ACTH basal secretion.

The literature on the mechanism of rapid glucocorticoid inhibition of HPA activity is confusing. In the rat, there is in vivo evidence that this involves an MR-mediated process (Atkinson et al., 2008), whereas ex vivo, high levels of corticosterone (10- to 20 -fold higher than binds classical MR) inhibitory responses sug- 
gest a less sensitive membrane-bound MR (Karst et al., 2005). At the level of the pituitary in vitro, however, rapid inhibition of membrane potential (Hua and Chen, 1989) and ACTH release (Dayanithi and Antoni, 1989; John et al., 2002; Buckingham et al., 2003) occurs via a nongenomic Annexin 1-mediated GR mechanism (Taylor et al., 1993). Our data reveal a response that is more rapid than demonstrated for Annexin 1 and fits better with recent in vitro and in vivo evidence for rapid corticosteroid processes acting via a membrane-bound GR (Tait et al., 2008; Breuner and Orchinik, 2009; Roy and Rai, 2009), probably mediated through a phosphatidylinositol 3-kinase pathway (Limbourg et al., 2002; Matthews et al., 2008; Roy and Rai, 2009). This suggests a fast feedback inhibition that targets the fusion of ACTH-containing vesicles with the cell membrane and their subsequent release from corticotrophs and explains why the constitutive release of POMC was unaffected.

It is of course possible that both GR and MR are involved in negative feedback, with GR effecting a rapid nongenomic feedback at the level of the anterior pituitary and MR sensing higher glucocorticoid levels while levels are still rising (Karst et al., 2005). These could exert different temporal dynamics and provide a feedback sensor mechanism with a wide range of sensitivity.

In conclusion, we provide novel evidence that HPA inhibitory feedback responses to corticosteroids occur within a very rapid time domain in man. This fast feedback inhibition is GR dependent, independent of $\mathrm{CRH}$ drive, occurs predominately at the level of the anterior pituitary, and only blocks the regulated pathway of ACTH release. Our ability to detect these robust rapid responses of HPA activity to exogenous corticosteroid feedback in man will allow us to investigate mechanisms underlying abnormal HPA activity in conditions such as depression, obesity, memory dysfunction, and aging.

\section{References}

Akaike H (1974) A new look at the statistical model identification. IEEE Trans Autom Control 19:716-723.

Atkinson HC, Wood SA, Castrique ES, Kershaw YM, Wiles CC, Lightman SL (2008) Corticosteroids mediate fast feedback of the rat hypothalamicpituitary-adrenal axis via the mineralocorticoid receptor. Am J Physiol Endocrinol Metab 294:E1011-E1022.

Boscaro M, Paoletta A, Scarpa E, Barzon L, Fusaro P, Fallo F, Sonino N (1998) Age-related changes in glucocorticoid fast feedback inhibition of adrenocorticotropin in man. J Clin Endocrinol Metab 83:1380-1383.

Boudinot FD, Jusko WJ (1986) Dose-dependent pharmacokinetics of prednisolone in normal and adrenalectomized rats. J Pharmacokinet Biopharm 14:453-467.

Breuner CW, Orchinik M (2009) Pharmacological characterization of intracellular, membrane, and plasma binding sites for corticosterone in house sparrows. Gen Comp Endocrinol 163:214-224.

Bright GM (1995) Corticosteroid-binding globulin influences kinetic parameters of plasma cortisol transport and clearance. J Clin Endocrinol Metab 80:770-775.

Buckingham JC, Solito E, John C, Tierney T, Taylor A, Flower R, Christian H, Morris J (2003) Annexin 1: a paracrine/juxtacrine mediator of glucorticoid action in the neuroendocrine system. Cell Biochem Funct 21:217-221.

Carnes M, Lent S, Feyzi J, Hazel D (1989) Plasma adrenocorticotropic hormone in the rat demonstrates three different rhythms within $24 \mathrm{~h}$. Neuroendocrinology 50:17-25.

Castro M, Elias LL, Elias PC, Moreira AC (2003) A dose-response study of salivary cortisol after dexamethasone suppression test in Cushing's disease and its potential use in the differential diagnosis of Cushing's syndrome. Clin Endocrinol (Oxf) 59:800-805.

Dallman MF, Yates FE (1969) Dynamic asymmetries in the corticosteroid feedback path and distribution-metabolism-binding elements of the adrenocortical system. Ann N Y Acad Sci 156:696-721.

Dayanithi G, Antoni FA (1989) Rapid as well as delayed inhibitory effects of glucocorticoid hormones on pituitary adrenocorticotropic hormone release are mediated by type II glucocorticoid receptors and require newly synthesized messenger ribonucleic acid as well as protein. Endocrinology 125:308-313.

De Kloet ER, Vreugdenhil E, Oitzl MS, Joëls M (1998) Brain corticosteroid receptor balance in health and disease. Endocr Rev 19:269-301.

Deuschle M, Schweiger U, Gotthardt U, Weber B, Körner A, Schmider J, Standhardt H, Lammers CH, Krumm B, Heuser I (1998) The combined dexamethasone/corticotropin-releasing hormone stimulation test is more closely associated with features of diurnal activity of the hypothalamopituitary-adrenocortical system than the dexamethasone suppression test. Biol Psychiatry 43:762-766.

Fehm HL, Voigt KH, Kummer G, Lang R, Pfeiffer EF (1979) Differential and integral corticosteroid feedback effects on ACTH secretion in hypoadrenocorticism. J Clin Invest 63:247-253.

Follenius M, Simon C, Brandenberger G, Lenzi P (1987) Ultradian plasma corticotropin and cortisol rhythms: time-series analyses. J Endocrinol Invest 10:261-266.

Grossmann C, Scholz T, Rochel M, Bumke-Vogt C, Oelkers W, Pfeiffer AF, Diederich S, Bahr V (2004) Transactivation via the human glucocorticoid and mineralocorticoid receptor by therapeutically used steroids in CV-1 cells: a comparison of their glucocorticoid and mineralocorticoid properties. Eur J Endocrinol 151:397-406.

Haller J, Mikics E, Makara GB (2008) The effects of non-genomic glucocorticoid mechanisms on bodily functions and the central neural system. A critical evaluation of findings. Front Neuroendocrinol 29:273-291.

Henley DE, Leendertz J, Russell GM, Wood SA, Taheri S, Woltersdorf W, Lightman S (2009) Development of an automated blood sampling system for use in humans. J Med Eng Technol 33:199-208.

Hua SY, Chen YZ (1989) Membrane receptor-mediated electrophysiological effects of glucocorticoid on mammalian neurons. Endocrinology 124:687-691.

Iranmanesh A, Lizarralde G, Veldhuis JD (1993) Coordinate activation of the corticotropic axis by insulin-induced hypoglycemia: simultaneous estimates of beta-endorphin, adrenocorticotropin and cortisol secretion and disappearance in normal men. Acta Endocrinol (Copenh) 128:521-528.

Jacobson L (2005) Hypothalamic-pituitary-adrenocortical axis regulation. Endocrinol Metab Clin North Am 34:271-292, vii.

John C, Cover P, Solito E, Morris J, Christian H, Flower R, Buckingham J (2002) Annexin 1-dependent actions of glucocorticoids in the anterior pituitary gland: roles of the $\mathrm{N}$-terminal domain and protein kinase C. Endocrinology 143:3060-3070.

Jones MT, Hillhouse EW, Burden JL (1977) Dynamics and mechanics of corticosteroid feedback at the hypothalamus and anterior pituitary gland. J Endocrinol 73:405-417.

Karst H, Berger S, Turiault M, Tronche F, Schütz G, Joëls M (2005) Mineralocorticoid receptors are indispensable for nongenomic modulation of hippocampal glutamate transmission by corticosterone. Proc Natl Acad Sci U S A 102:19204-19207.

Keenan DM, Licinio J, Veldhuis JD (2001) A feedback-controlled ensemble model of the stress-responsive hypothalamo-pituitary-adrenal axis. Proc Natl Acad Sci U S A 98:4028-4033.

Keenan DM, Roelfsema F, Biermasz N, Veldhuis JD (2003) Physiological control of pituitary hormone secretory-burst mass, frequency, and waveform: a statistical formulation and analysis. Am J Physiol Regul Integr Comp Physiol 285:R664-R673.

Keenan DM, Chattopadhyay S, Veldhuis JD (2005) Composite model of time-varying appearance and disappearance of neurohormone pulse signals in blood. J Theor Biol 236:242-255.

Keller-Wood ME, Dallman MF (1984) Corticosteroid inhibition of ACTH secretion. Endocr Rev 5:1-24.

Limbourg FP, Huang Z, Plumier JC, Simoncini T, Fujioka M, Tuckermann J, Schütz G, Moskowitz MA, Liao JK (2002) Rapid nontranscriptional activation of endothelial nitric oxide synthase mediates increased cerebral blood flow and stroke protection by corticosteroids. J Clin Invest 110:1729-1738.

Maddock C, Pariante CM (2001) How does stress affect you? An overview of stress, immunity, depression and disease. Epidemiol Psichiatr Soc 10:153-162.

Matthews L, Berry A, Ohanian V, Ohanian J, Garside H, Ray D (2008) Caveolin mediates rapid glucocorticoid effects and couples glucocorti- 
coid action to the antiproliferative program. Mol Endocrinol 22:13201330.

Moore CR, Price D (1932) Gonad hormone functions, and the reciprocal influence between gonads and hypophysis with its bearing on the problem of sex hormone antagonism. Am J Anat 50:13-71.

Pariante CM, Miller AH (2001) Glucocorticoid receptors in major depression: relevance to pathophysiology and treatment. Biol Psychiatry 49: 391-404.

Pichler R, Crespillo C, Maschek W, Esteva I, Soriguer F, Sfetsos K, Auböck J (2004) Plasma levels of alpha-melanotropin and ACTH-like immunoreactivities do not vary by season or skin type in women from southern and central Europe. Neuropeptides 38:325-330.

Pincus SM, Keefe DL (1992) Quantification of hormone pulsatility via an approximate entropy algorithm. Am J Physiol 262:E741-E754.

Posener JA, Schildkraut JJ, Williams GH, Schatzberg AF (1997) Cortisol feedback effects on plasma corticotropin levels in healthy subjects. Psychoneuroendocrinology 22:169-176.

Pritchard LE, White A (2007) Neuropeptide processing and its impact on melanocortin pathways. Endocrinology 148:4201-4207.

Raff H (2008) Immulite vs Scantibodies IRMA plasma ACTH assay. Clin Chem 54:1409-1410.

Reader SC, Alaghband-Zadeh J, Daly JR, Robertson WR (1982) Negative rate-sensitive feedback effects on adrenocorticotrophin secretion by cortisol in normal subjects. J Endocrinol 92:443-448.

Roberts JL, Chen CL, Eberwine JH, Evinger MJ, Gee C, Herbert E, Schachter BS (1982) Glucocorticoid regulation of proopiomelanocortin gene expression in rodent pituitary. Recent Prog Horm Res 38:227-256.

Robinson DS, Campbell DA, Durham SR, Pfeffer J, Barnes PJ, Chung KF (2003) Systematic assessment of difficult-to-treat asthma. Eur Respir J 22:478-483.

Rose JQ, Yurchak AM, Jusko WJ (1981) Dose dependent pharmacokinetics of prednisone and prednisolone in man. J Pharmacokinet Biopharm 9:389-417.

Rousseau K, Kauser S, Pritchard LE, Warhurst A, Oliver RL, Slominski A, Wei ET, Thody AJ, Tobin DJ, White A (2007) Proopiomelanocortin (POMC), the ACTH/melanocortin precursor, is secreted by human epidermal keratinocytes and melanocytes and stimulates melanogenesis. FASEB J 21:1844-1856.

Roy B, Rai U (2009) Genomic and non-genomic effect of cortisol on phagocytosis in freshwater teleost Channa punctatus: an in vitro study. Steroids 74:449-455.

Sayers G, Portanova R (1974) Secretion of ACTH by isolated anterior pituitary cells: kinetics of stimulation of corticotropin-releasing factor and of inhibition by corticosterone. Endocrinology 94:1723-1730.

Schmider J, Lammers CH, Gotthardt U, Dettling M, Holsboer F, Heuser IJ (1995) Combined dexamethasone/corticotropin-releasing hormone test in acute and remitted manic patients, in acute depression, and in normal controls. I. Biol Psychiatry 38:797-802.
Son GH, Chung S, Choe HK, Kim HD, Baik SM, Lee H, Lee HW, Choi S, Sun W, Kim H, Cho S, Lee KH, Kim K (2008) Adrenal peripheral clock controls the autonomous circadian rhythm of glucocorticoid by causing rhythmic steroid production. Proc Natl Acad Sci U S A 105:2097020975.

Stavreva DA, Wiench M, John S, Conway-Campbell BL, McKenna MA, Pooley JR, Johnson TA, Voss TC, Lightman SL, Hager GL (2009) Ultradian hormone stimulation induces glucocorticoid receptor-mediated pulses of gene transcription. Nat Cell Biol 11:1093-1102.

Tait AS, Butts CL, Sternberg EM (2008) The role of glucocorticoids and progestins in inflammatory, autoimmune, and infectious disease. J Leukoc Biol 84:924-931.

Taylor AD, Cowell AM, Flower J, Buckingham JC (1993) Lipocortin 1 mediates an early inhibitory action of glucocorticoids on the secretion of ACTH by the rat anterior pituitary gland in vitro. Neuroendocrinology $58: 430-439$.

Ulrich-Lai YM, Arnhold MM, Engeland WC (2006) Adrenal splanchnic innervation contributes to the diurnal rhythm of plasma corticosterone in rats by modulating adrenal sensitivity to ACTH. Am J Physiol Regul Integr Comp Physiol 290:R1128-R1135.

Veldhuis JD, Iranmanesh A, Lizarralde G, Johnson ML (1989) Amplitude modulation of a burstlike mode of cortisol secretion subserves the circadian glucocorticoid rhythm. Am J Physiol 257:E6-E14.

Veldhuis JD, Iranmanesh A, Johnson ML, Lizarralde G (1990) Twentyfour-hour rhythms in plasma concentrations of adenohypophyseal hormones are generated by distinct amplitude and/or frequency modulation of underlying pituitary secretory bursts. J Clin Endocrinol Metab 71:1616-1623.

Veldhuis JD, Johnson ML, Veldhuis OL, Straume M, Pincus SM (2001) Impact of pulsatility on the ensemble orderliness (approximate entropy) of neurohormone secretion. Am J Physiol Regul Integr Comp Physiol 281:R1975-R1985.

Walker JJ, Terry J, Lightman SL (2010) Origin of ultradian activity in the hypothalamic-pituitary-adrenal axis. Proc Biol Sci. Advance online publication. Retrieved February 3, 2010. doi:10.1098/rspb.2009.2148.

Watts AG (2005) Glucocorticoid regulation of peptide genes in neuroendocrine CRH neurons: a complexity beyond negative feedback. Front Neuroendocrinol 26:109-130.

Windle RJ, Wood SA, Shanks N, Lightman SL, Ingram CD (1998) Ultradian rhythm of basal corticosterone release in the female rat: dynamic interaction with the response to acute stress. Endocrinology 139:443-450.

Young EA, Abelson J, Lightman SL (2004) Cortisol pulsatility and its role in stress regulation and health. Front Neuroendocrinol 25:69-76.

Zimmermann E, Critchlow V (1969) Negative feedback and pituitary-adrenal function in female rats. Am J Physiol 216:148-155.

Zimmermann E, Critchlow V (1972) Short latency suppression of pituitaryadrenal function within physiological levels of corticosterone in the female rat. Neuroendocrinology 9:235-243. 